\title{
Development of parallel auditory thalamocortical pathways for two different behaviors
}

\author{
Khaleel A. Razak ${ }^{1}$ and Zoltan M. Fuzessery ${ }^{2 *}$ \\ ' Department of Psychology, University of California, Riverside, CA, USA \\ 2 Department of Zoology and Physiology, University of Wyoming, Laramie, WY, USA
}

\section{Edited by:}

Enrique Saldaña, Universidad de Salamanca, Spain

Reviewed by:

Andrew J. King, University of Oxford, UK

Douglas C. Fitzpatrick, The University of North Carolina at Chapel Hill, USA

\section{${ }^{*}$ Correspondence:}

Zoltan M. Fuzessery, Department of Zoology and Physiology, University of Wyoming, 1000 East University

Avenue, Laramie, WY 82071 USA.

e-mail:zmf@uwyo.edu
Auditory thalamocortical connections are organized as parallel pathways that originate in different divisions of the medial geniculate body (MGB). These pathways may be involved in different functions. Surprisingly little is known about the development of these connections. Here we review studies of the organization and development of auditory thalamocortical pathways in the pallid bat. The pallid bat depends primarily on passive hearing of prey-generated noise for localizing prey, while reserving echolocation for general orientation and obstacle avoidance. In the inferior colliculus (IC) and the auditory cortex, physiological studies show that noise and echolocation calls are processed in segregated regions. Injection of retrograde tracers in physiologically characterized cortical sites show that the ventral division of the MGB (MGBv) projects to the cortical region selective for noise. The cortical region selective for echolocation calls receives input from the suprageniculate (SG) nucleus in the dorsal MGB, but not from the MGBv. Taken together, these studies reveal parallel IC-MGB-cortex pathways involved in echolocation and passive listening. There is overlap of thalamocortical pathways during development. At 2-weeks postnatal, when the bat begins to exhibit adult-like hearing thresholds, the SG projects to both noise- and echolocation call-selective regions. The MGBv, as in adults, projects only to the noise-selective region. The connections become adult-like only after 2-months postnatal. These data suggest that parallel auditory thalamocortical pathways may segregate in an experiencedependent fashion, a hypothesis that remains to be tested in any species.

Keywords: auditory cortex, medial geniculate body, auditory development, plasticity, parallel pathways, echolocation, thalamocortical

\section{PARALLEL AUDITORY THALAMOCORTICAL PATHWAYS}

A hallmark of sensory system organization is parallel pathways. In the auditory system, auditory nerve fibers diverge into different divisions of the cochlear nucleus setting the stage for multiple ascending pathways. At the thalamocortical level, parallel pathways arise from three distinct divisions of the medial geniculate body (MGB; for review see Winer et al., 2005). These parallel pathways are purported to serve different functions in hearing. Thalamocortical connections include a tonotopic pathway through the MGBv (ventral division) which forms the cortical substrate for functional subregions within isofrequency contours (reviewed in Read et al., 2002). The pathway through the MGBm (medial division) is implicated in multisensory integration and learning (Wepsic, 1966; Edeline and Weinberger, 1992; Bordi and LeDoux, 1994; for review see $\mathrm{Hu}, 2003)$. The non-tonotopic pathway through the MGBd (dorsal division, Raczkowski et al., 1976; Andersen et al., 1980; Morel and Imig, 1987; for review see Imig and Morel, 1983; Rouiller, 1997) may be involved in representing complex sounds (Aitkin and Dunlop, 1968).

The hypothesis that parallel thalamocortical pathways are involved in different aspects of hearing is supported by studies in three species of bats from different families: the mustached bat (Pteronotus parnellii; Mormoopidae family), the horseshoe bat (Rhinolophus rouxi; Rhinolophidae family) and the pallid bat (Antrozous pallidus; Vespertilionidae family). The mustached bat auditory cortex contains a primary auditory cortex (A1) with a tonotopic map (reviewed in Suga, 1989). This map, as in all other species examined, receives input from the MGBv. Some specializations for echolocation, such as over-representation of dominant harmonic frequencies of the echolocation call, are present in this pathway, but within the context of tonotopic representation. Information relevant to target distance is represented by delaytuned combination-sensitive neurons. A large delay-tuned area is located dorsal to A1. Smaller delay-tuned areas are present ventral to A1. Injections of different tracers in different delay-tuned areas label separate regions in the MGB indicating that multiple delay-tuned areas are present in the thalamus (Pearson et al., 2007; also see Wenstrup, 1999 for physiological studies with a similar conclusion) and that each thalamic delay-tuned area representation projects independently, and in parallel, to a cortical delaytuned area (Pearson et al., 2007). Particularly, labeling was found in the rostral pole nucleus. Although it remains debated whether the rostral pole nucleus should be part of the dorsal division, the lateral part of the posterior group or an extension of the ventral division (Morel and Imig, 1987; Wenstrup et al., 1994; Lee et al., 2004; Pearson et al., 2007), it is clear there are parallel pathways conveying information from the MGB to the cortex.

In the horseshoe bat, the cortical region termed dorsal cortex contains neurons that may be involved in target ranging. These neurons receive input from the MGBd (Radtke-Schuller, 2004; 
Radtke-Schuller et al., 2004). The primary auditory cortex receives input from the MGBv. In both the mustached and horseshoe bat the suprageniculate (SG) nucleus, a part of the MGBd, projects diffusely to the entire auditory cortex, with a significant input to non-primary cortical regions involved in processing echolocation calls. Taken together, these data indicate that the thalamocortical pathways that serve fine frequency analysis and target distance calculation in the mustached and horseshoe bat are mostly segregated(Figure 1A).

Studies on the pallid bat provide evidence that parallel auditory pathways may represent sounds used in two different behaviors. The pallid bat localizes terrestrial prey by listening to prey-generated noise while reserving echolocation calls for general orientation and obstacle avoidance (Bell, 1982; Fuzessery et al., 1993). For echolocation, it uses a downward frequency modulated (FM) sweep $(60 \rightarrow 30 \mathrm{kHz})$. For prey localization it depends on noise transients $(5-40 \mathrm{kHz})$. Its auditory cortex and inferior colliculus (IC) are organized to process FM sweeps and noise in mostly segregated regions (Fuzessery, 1994; Razak and Fuzessery, 2002). In the auditory cortex, there is a tonotopic map with frequencies from 5 to $70 \mathrm{kHz}$. Most neurons with tuning between 5 and $30 \mathrm{kHz}$ respond best to noise transients. This region has been termed the low-frequency region (LFR). Most neurons with tuning between 30 and $60 \mathrm{kHz}$ respond best to downward FM sweeps. This region has been termed the high-frequency region (HFR).

The majority of inputs to the LFR and HFR in adult pallid bats arise from different divisions of the MGB (Figure 2, schematized in Figure 1B). Placement of retrograde tracers in the LFR labeled neurons predominantly in the MGBv with no label in the SG (Razak et al., 2007). Placement of tracers in the HFR labeled neurons in the SG, but not the MGBv. The MGBm and parts of MGBd outside of the SG send minor projections to both the LFR and the HFR. Thus parallel thalamocortical pathways represent sounds involved in two different behaviors, with the SG $\rightarrow$ HFR connections putatively involved in echolocation behavior and $\mathrm{MGBv} \rightarrow$ LFR connections putatively involved with passive prey localization.

\section{A SIGNIFICANT GAP IN KNOWLEDGE: DEVELOPMENT OF AUDITORY THALAMOCORTICAL CONNECTIONS}

While the existence and functional significance of parallel thalamocortical pathways are established, very little is known about the development of these pathways. The development of these pathways between the MGB and the auditory cortex has been the subject of only one study. Gurung and Fritzsch (2004) found that the MGB innervates the auditory cortex before onset of patterned sensory input. However, whether segregation of MGB-auditory cortex inputs into parallel pathways depends on sensory experience remains unknown. Research on the development of auditory pathways has focused on connections below the thalamus. Studies of connections of the midbrain (Friauf and Kandler, 1990; Gabriele et al., 2000, 2007) and lower brainstem (Leake et al., 2002) suggest that the nucleotopic connections are mostly adult-like before hearing onset. Postnatal refinement or maintenance of connections, perhaps in an activity-dependent manner, is restricted to sharpening tonotopic organization (Kim and Kandler, 2003; Franklin et al., 2006; Leake et al., 2006) and fine tuning bilateral inputs within nuclei involved in sound localization (Kapfer et al., 2002).

\section{DEVELOPMENT OF PARALLEL THALAMOCORTICAL CONNECTIONS IN THE PALLID BAT}

The pallid bat is suited to address the development of thalamocortical connections for several reasons. First, as mentioned above, parallel pathways can be linked to representation of different behaviorally relevant sounds. Second, the ontogeny of both echolocation

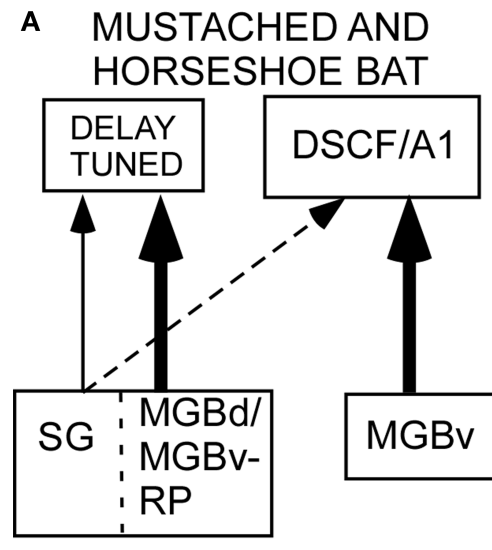

FIGURE 1 | Schematic of thalamocortical pathways in adult mustached, horseshoe and pallid bats and young pallid bat. (A) In the horseshoe bat, the MGBd projects mainly to regions dorsal to A1 that contain the combinationsensitive neurons used in target distance computation. The MGBv projects to A1. In the mustached bat, the rostral pole nucleus projects to the delay-tuned areas. It is not clear if the rostral pole nucleus should be considered a part of the ventral or dorsal MGB. Therefore, the term "MGBd/MGBv-RP" is used in this schematic. In both species, the SG, considered a part of the MGBd, projects diffusely but more to the non-primary cortex than primary cortex. (B) In adult
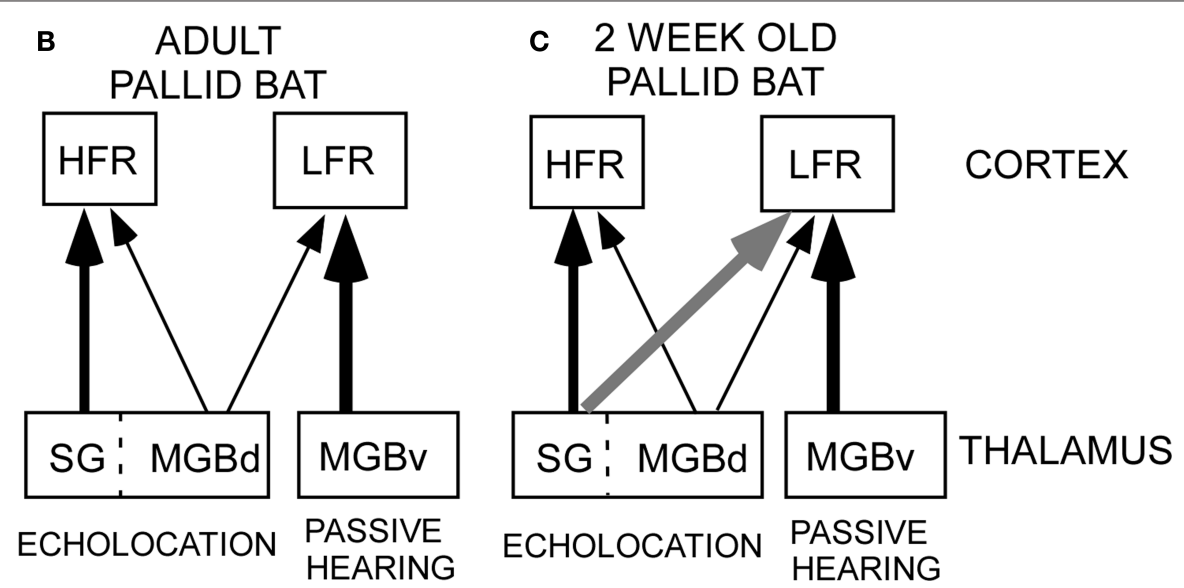

pallid bats, the HFR involved in echolocation behavior receives input from the SG and the MGBd, but not the MGBv. The LFR, involved in passive localization, receives input from the $M G B v$, but not the $S G$. Based on response selectivity in the auditory cortex, the SG $\rightarrow$ HFR pathway is involved in echolocation behavior while the MGBv $\rightarrow$ LFR pathway is involved in passive localization of preygenerated noise. (C) In a 2-week-old pallid bat pup, however, the pathways overlap. This is because the SG projects to both the LFR and HFR. The pup MGBv, as in adults, does not project to the HFR. Thus anatomically segregated pathways arise through postnatal refinement of initially overlapping connections. 


\section{ADULT PALLID BAT}

\section{HFR INJECTION}

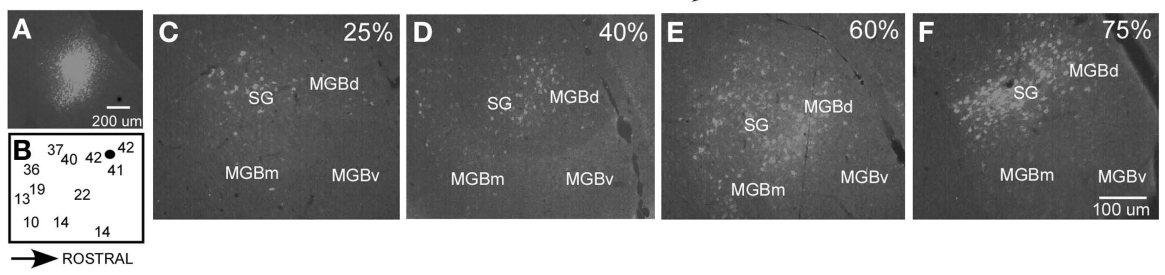

\section{LFR INJECTION}
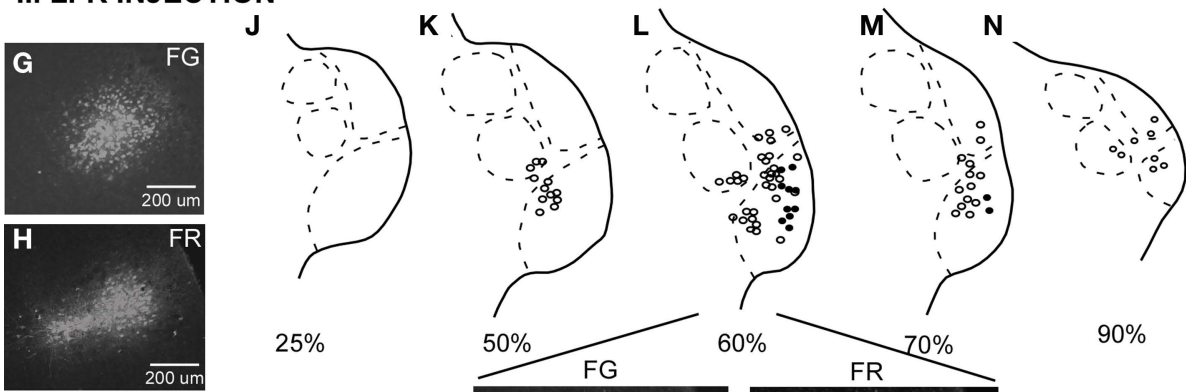

$25 \%$
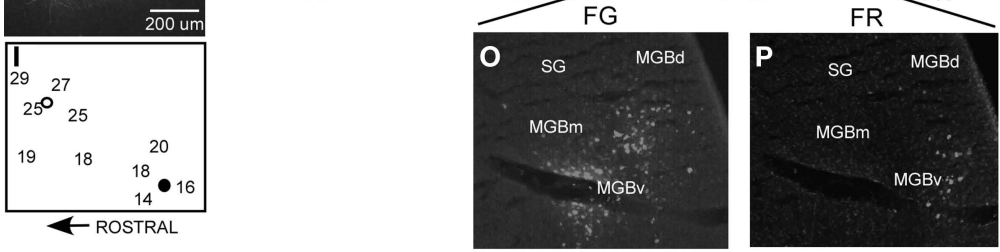

\section{DUAL INJECTION}

Q

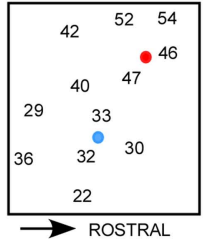

$\mathbf{R}$

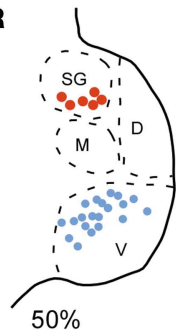

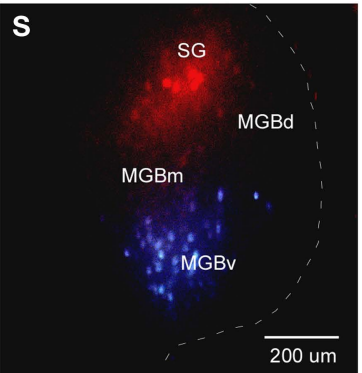

FIGURE 2 | Parallel thalamocortical pathways in the adult pallid bat auditory system. I (A-F) An example of injection made in the HFR.

(A) Fluorogold (FG) injection site. (B) Frequency selectivity of injection site. The injection was made near neurons tuned $\sim 42 \mathrm{kHz}$ and selective for downward FM sweeps. (C-F) Photomicrographs of increasingly more rostral locations of the MGB show that labeled neurons were found in the SG, MGBm, and MGGd, but not in the MGBv. II (G-P) An example of injections made in the LFR. (G) FG injection site (H) Fluororuby (FR) injection site. (I) FG was injected near neurons tuned $\sim 26 \mathrm{kHz}$. FR was injected near neurons tuned $\sim 15 \mathrm{kHz}$. (J-N) Schematics of increasingly rostral sections through the MGB showing that labeled neurons were found in the MGBv, MGBm, and MGBd, but not the SG. (O,P)

Photomicrographs showing FG and FR labeled cells near the rostrocaudal center of the MGB. III (Q-S) An experiment in which FG was injected in the LFR and FR was injected in the HFR. (Q) Schematic of injection sites. (R) Schematic of a section at the rostrocaudal center of the MGB showing that FG labeled neurons were present in MGBv, but not the SG, and that FR labeled cells were found in the SG, but not the MGBv. (S) Photomicrograph of the section shown in $(\mathbf{R})$ behavior (Brown et al., 1978) and functional organization of auditory cortex (Razak and Fuzessery, 2007) has been studied. This provides a behavioral framework during development to compare anatomical and physiological data. The overall functional organization of auditory cortex, including frequency representation, is adult-like early in development (Razak and Fuzessery, 2002, 2007).
An exception is the prevalence and anatomical distribution of functionally bimodal neurons (Razak et al., 1999). These neurons appear to receive input from both pathways, and have two discrete tuning curves tuned to frequencies used in echolocation and passive sound localization. In adults, these bimodal neurons are found near the interface of the LFR and HFR. In pups, however, they are 
more widely distributed (Razak and Fuzessery, 2007), suggesting that projections from the MGBv and SG may show greater overlap in their cortical targets. We tested this hypothesis by retrograde tracing of MGB inputs from physiologically identified injection sites in the LFR and HFR of auditory cortex.

Details about injection method, sizes, and definition of divisions of the MGB have been addressed in detail in the original papers (Razak and Fuzessery, 2007, 2009). Although it was relatively easy to distinguish the SG from rest of the MGBd and MGBv, the boundary between MGBd and MGBv was less obvious based on Nissl or neutral red stains. However, luxol fast blue, neutral red stained sections show that MGBv stains weakly for myelin, whereas the MGBd contains fibers that run in a dorsomedial to ventrolateral direction. The boundary between the dorsal and the ventral divisions was drawn based on myelin staining. The injection sizes are noted in Razak and Fuzessery (2007). A comparison of adult and pup brain photomicrographs shows similar injection sizes for fluroruby and flurogold (Figures 2 and 3 ).

There is greater overlap in thalamocortical connections during early development (Razak et al., 2009). Injections of retrograde tracers in the LFR labeled neurons in both the MGBv and the SG (Figure 3, schematic in Figure 1C). The input from the SG to LFR was prominent from the caudal half of the MGB, and was at least as strong as the input from the MGBv to the LFR based on the number of labeled neurons. However, as in adults, there was no input from the pup MGBv to the HFR. An overlap of pathways was seen in P60 bats, but not in P150 bats, indicating that the refinement is occurring during this time period. Pallid bats obtain adult-like hearing sensitivity $\sim$-weeks postnatal. Pallid bats begin to fly and use echolocation in flight around 5-6 weeks (Brown et al., 1978) and are fully weaned around 12 weeks. Thus the refinement occurs a few weeks after flight onset, and more than 6 weeks after onset of adult-like hearing. These data show that the parallel thalamocortical pathways seen in adults emerge during postnatal development and may involve refinement of overlapping connections well after the bat begins to experience patterned input.

\section{WHY PARALLEL PATHWAYS FOR NOISE AND FM SWEEPS?}

We have suggested that the parallel pathways for noise and FM sweep processing observed in the IC, MGB, and cortex are an adaptation for gleaning behavior in the pallid bat (Barber et al., 2003). As the bat hunts, it receives both echoes from the flight path and prey-generated noise from the ground. The bat has to segregate these two streams of temporally overlapping inputs. The noise and FM sweeps used in prey localization and echolocation differ in spectral (low versus high frequency), temporal (noise versus sweep), and spatial (ground versus flight path) qualities. These differences should enable the bat to segregate the two sounds (Bregman, 1990). In addition, the parallel processing with strong filters (noise versus FM sweep selectivity) will provide a substrate for additional segregation of the two auditory streams. During development, the onset of adult-like hearing thresholds may not be the critical experience for initiation of segregated pathways. The experience with dual processing of echoes and terrestrial prey-generated noise may be more important in initiating segregated pathways; therefore refinement occurs when the bat begins to hunt. Such experiencedependent plasticity has been described previously in the barn owl midbrain (Bergan et al., 2005).

\section{A NOTE ON THE SUPRAGENICULATE NUCLEUS}

The SG appears to be a phylogenetically and ontogentically malleable region of the thalamus. In non-chiropterans the SG is dominated by visual input from the superior colliculus (cat: Calford and Aitkin, 1983; Katoh and Benedek, 1995; rat: Tanaka et al., 1985). Less than $15 \%$ of the neurons respond only to auditory stimuli, whereas $65 \%$ of the SG neurons respond to visual stimuli alone (Benedek et al., 1997). The entire rostrocaudal extent of the SG receives input from the superior colliculus (SC) (Katoh and Benedek, 1995). Auditory inputs to the SG may arise from the nucleus of the central acoustic tract in the brainstem and the external nucleus of the IC (Henkel, 1983; Katoh and Benedek, 1995), both of which are considered to be a part of the extralemniscal auditory pathways. The SG projects primarily to non-primary auditory cortical areas, although sparse connections to the primary auditory cortex are found in many species (owl monkey: Morel and Kaas, 1992; macaque monkey: Hackett et al., 1998; tamarin: Luethke et al., 1989; dog: Malinowska and Kosmal, 2003; rat: Roger and Arnault, 1989).

In bats, the SG is dominated by auditory inputs. The SG of the mustached bat, unlike non-chiropterans, receives inputs from all frequency bands of the central nucleus of the IC (Wenstrup et al., 1994). Like non-chiropterans, the SG also receives direct input from the nucleus of the central acoustic tract (Casseday et al., 1989; Gordon and O'Neill, 2000). In addition to broad regions of the auditory cortex, the SG of the mustached bat projects to frontal cortex, which in turn projects to the SC, suggesting a role in acoustic-motor reflexes (Kobler et al., 1987). In the horseshoe bat, the SG projects to both primary and non-primary cortical fields (Radtke-Schuller et al., 2004). There is overlap in projections from the SG and the MGBv to any given cortical region, but the dorsal cortical fields (non-primary) receive most SG inputs. In the pallid bat, the echolocation pathway is primarily routed through the SG. Taken together, these studies suggest that the SG can be taken over to increase thalamic representation of species-specific dominant sensory modalities.

The developmental plasticity of SG connections is illustrated by cross-modal plasticity in ferrets (Pallas et al., 1990). By appropriate lesions of retinal axon targets, visual input can be guided to the MGB. This rewiring leaves the MGBv-primary auditory connections similar to those of controls. However, an anomalous input to the primary auditory cortex arises from the dorsal thalamus, including the SG, in the cross-modal animals. This finding suggests that the SG typically receives and sends more exuberant connections during early development. These connections may be either stabilized or pruned by activity-dependent mechanisms. The data from the pallid bat thalamocortical development support the hypothesis that the SG sends exuberant projections during early development.

\section{FUTURE STUDIES}

1. The studies on pallid bat thalamocortical connections suggest postnatal refinement. However, it remains unclear if this is experience-dependent. It is possible to modify echolocation experience during development (Razak et al., 2008). Such modifications significantly alter response selectivity within the 


\section{PALLID BAT PUP}

\section{LFR INJECTION (P15)}
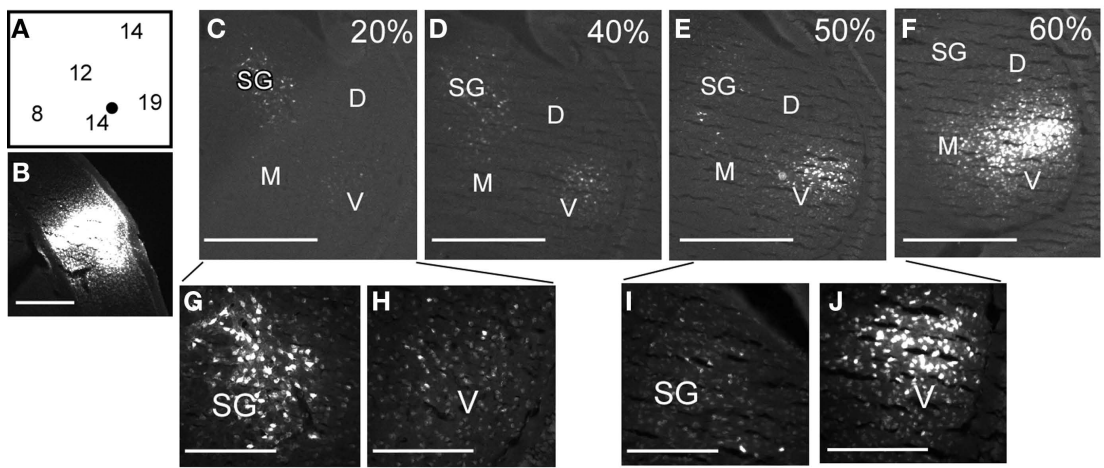

II. HFR INJECTION (P20)
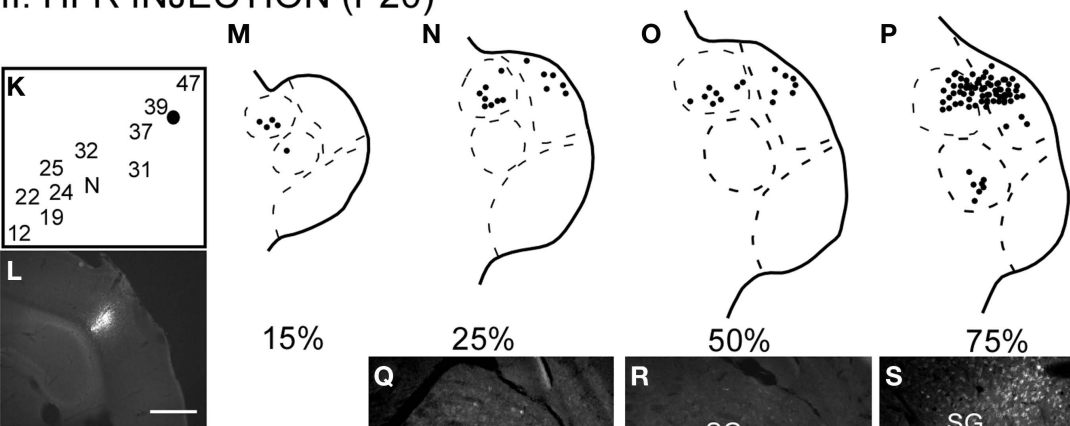

$15 \%$
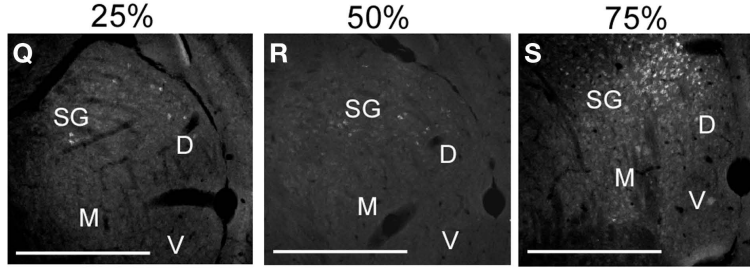

III. DUAL INJECTIONS (P30)
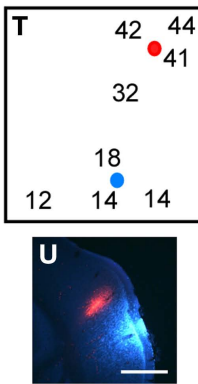

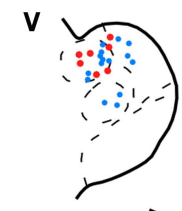

$15 \%$

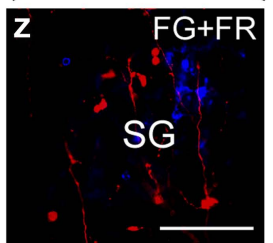

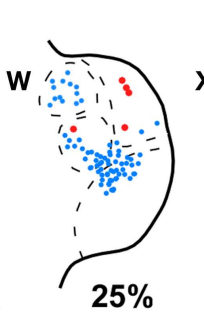

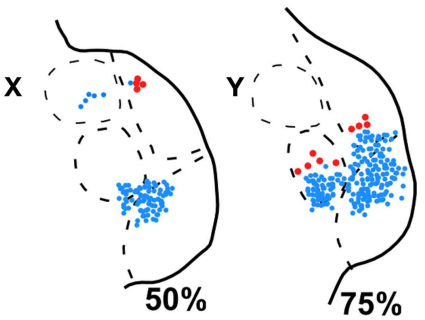

FIGURE 3 |The thalamocortical pathways overlap during early development. This occurs because the SG projects to both LFR and HFR in pups. I (A-J) LFR injection in a P15 pup. (A) FG was injected in the LFR near sites with tuning $\sim 15 \mathrm{kHz}$. (B) FG injection site. (C-F) Photomicrographs of increasingly rostral sections through the MGB demonstrate that both the SG and MGBv show labeled cells. (G,H) Magnified view of the SG and (I,J) MGBv show strong label in both areas. It can also be noted that caudal SG sends more projections to the LFR in pups than rostral SG. II (K-S) HFR injection in a P20 pup. (K) The injection site had best frequencies near $40 \mathrm{kHz}$. (L) FG injection site. (M-P) Schematic and (Q-S) Photomicrographs of the MGB show that labeled cells were present in the SG and MGBd, but not the MGBv. III (T-Z) Injections in both LFR and HFR. (T) FG was injected in the LFR. FR was injected in the HFR. (U) Injection sites. (V-Y) FG and FR labeled cells were seen in the SG, while only FG labeled cells were seen in the MGBv. (Z) Magnified view of the SG shows FG and FR labeled cells, although no double labeled cells were seen. Scale bars: $500 \mu \mathrm{m}$ in B-F; $200 \mu \mathrm{m}$ in $\mathbf{G}-\mathbf{J}$. 
echolocation pathway. Future studies will address how refinement of pathways proceeds in bats with altered experience with echolocation calls.

2. Although several studies have focused on development of auditory pathways below the thalamus, the pallid bat is the only animal we are aware of in which postnatal development of auditory thalamocortical connections has been examined. Therefore it remains unclear if the postnatal refinement of parallel thalamocortical pathways is a general phenomenon. In animals, such as the cat, in which the adult auditory pathways have been well characterized, studies are required on the development of these pathways. Significantly more is known about development of visual and somatosensory thalamocortical pathways.

3. A key hypothesis to emerge based on physiological and anatomical studies of the pallid bat auditory system is that in gleaning bats, parallel pathways from the IC-MGB-cortex represent different sounds used in different behaviors. This hypothesis is supported by physiological studies in the IC of Megaderma lyra, another gleaning bat from a different family (Rubsamen et al., 1988). Gleaning as a hunting strategy appears to have evolved independently in different families of bats. If parallel pathways are an adaptation for gleaning in the pallid bat, then a similar organization of the auditory system must be present in other gleaning bats. This hypothesis of convergent evolution of the auditory system remains to be tested. One unusual aspect of

\section{REFERENCES}

Aitkin, L. M., and Dunlop, C. W. (1968). Interplay of excitation and inhibition in the cat medial geniculate body. $J$. Neurophysiol. 31, 44-61.

Andersen, R. A., Knight, P. J., and Merzenich, M.M. (1980). The thalamocortical and corticothalamic connections $\mathrm{AI}, \mathrm{AII}$, and the anterior auditory field (AAF) in the cat: Evidence for two largely segregated systems of connections. J. Comp. Neurol. 194, 663-701.

Barber, J. R., Razak, K. A., and Fuzessery, Z. M. (2003). Can two streams of auditory information be processed simultaneously? Evidence from the gleaning bat Antrozous pallidus. $J$. Comp. Physiol. A 189, 843-855.

Bell, G. P. (1982). Behavioral and ecological aspects of gleaning by the desert insectivorous bat, Antrozous pallidus (Chiroptera:Vespertilionidae). Behav. Ecol. Sociobiol. (Print) 10, 217-223.

Benedek, G., Pereny, J., Kovacs, G., FischerSzatmari, L., and Katoh, Y. Y. (1997). Visual, somatosensory, auditory and nociceptive modality properties in the feline suprageniculate nucleus. Neuroscience 78, 179-189.

Bergan, J.F., Ro, P., Ro, D., and Knudsen, E. I. (2005). Hunting increases adaptive auditory map plasticity in adult barn owls. J. Neurosci. 25, 9816-9820.

Bordi, F., and LeDoux, J. E. (1994). Response properties of single units that project to the amygdala. II. Cells receiving convergent auditory and somatosensory inputs and cells antidromically activated by amygdala stimulation. Exp. Brain Res. 98, 275-286.

Bregman, A. S. (1990). Auditory Scene Analysis. Cambridge, MA: MIT Press.

Brown, P., Grinnell, A. D., and Harrison, J. (1978). The development of hearing in the pallid bat, Antrozous pallidus. J. Comp. Physiol. A 126, 169-182.

Calford, M. B., and Aitkin, L. M. (1983). Ascending projections to the medial geniculate body of the cat: Evidence for multiple, parallel auditory pathways through thalamus. J. Neurosci. 3, 2365-2380.

Casseday, J. H., Kobler, J. B., Isbey, S. F., and Covey, E. (1989). Central acousextralemniscal auditory pathway to the thalamus. J. Comp. Neurol. 287, 247-259.

Edeline, J.-M., and Weinberger, N. M. (1992). Associative retuning in the thalamic source of input to the amygdala and auditory cortex: Receptive field plasticity in the medial division of the medial geniculate body. Behav. Neurosci. 106, 81-105.

Franklin, S. R., Brunso-Bechtold, J. K., and Henkel, C. K. (2006). Unilateral in areas of rat auditory thalamus tic tract in an echolocating bat: An

the pallid bat thalamocortical connections is that the cortical tonotopic map is composed of non-overlapping inputs from two different divisions of the MGB (Razak et al., 2007). The presence of such an organization in other gleaners will strongly suggest a modified thalamocortical plan in gleaners compared to other mammals, including obligate echolocating bats.

4. The molecular mechanisms that make the SG ontogenetically and phylogentically labile are unknown. Recent studies that compared FOXP2 expression between echolocating and non-echolocating bats identified the SG as one of the nuclei in which expression was higher in the echolocators (Berquist et al., SFN abstracts, 2009). Expression of FOXP2 in the MGB also changes in an activity-dependent manner (Horng et al., 2009). FOXP2 may therefore be a part of the molecular milieu of the SG that makes it developmentally and evolutionarily more labile. Future studies comparing the expression of FOXP2 across various divisions of the MGB will be useful in determining the mechanisms of SG malleability and to address mechanisms underlying connectional and therefore functional plasticity in the thalamocortical connections.

\section{ACKNOWLEDGMENTS}

We thank T. Zumsteg for valuable comments on the manuscript and G. McLellan for programming the software required for this study. This research was supported by an NIDCD grant DC05202 and INBRE P20 RR0 16474-04.

cochlear ablation before hearing onset disrupts the maintenance of dorsal nucleus of the lateral lemniscus projection patterns in the rat inferior colliculus. Neuroscience 143, 105-115.

Friauf,E., and Kandler, K. (1990).Auditory projections to the inferior colliculus of the rat are present by birth. Neurosci. Lett. 120, 58-61.

Fuzessery, Z. M. (1994). Response selectivity for multiple dimensions of frequency sweeps in the pallid bat inferior colliculus. J. Neurophysiol. 72, 1061-1079.

Fuzessery, Z. M., Buttenhoff, P., Andrews, B., and Kennedy, J. M. (1993). Passive sound localization of prey by the pallid bat (Antrozous p. pallidus). J. Comp Physiol. [A] 171, 767-777.

Gabriele, M.L., Brunso-Bechtold,J.K., and Henkel, C. K. (2000). Development of afferent patterns in the inferior colliculus of the rat: Projection from the dorsal nucleus of the lateral lemniscus. J. Comp. Neurol. 416, 368-382.

Gabriele, M. L., Shahmoradian, S. H., French, C. C., Henkel, C. K., and McHaffie, J. G. (2007). Early segregation of layered projections from the lateral superior olivary nucleus to the central nucleus of the inferior colliculus in the neonatal cat. Brain Res. $1173,66-77$.

Gordon, M., and O'Neill, W. E. (2000). An extralemniscal component of the mustached bat inferior colliculus selective for direction and rate of linear frequency modulations. J. Comp. Neurol. 426, 165-181.

Gurung, B., and Fritzsch, B. (2004). Time course of embryonic midbrain and thalamic auditory connection development in mice as revealed by carbocyanine dye tracing. J. Comp. Neurol. 479, 309-327.

Hackett, T. A., Stepniewska, I., and Kaas, J.H. (1998). Thalamocortical connections of the parabelt auditory cortex in macaque monkeys. J. Comp. Neurol. 400, 271-286.

Henkel, C. K. (1983). Evidence of subcollicular auditory projections to the medial geniculate nucleus in the cat: An autoradiographic and horseradish peroxidase study. Brain Res. 259, 21-30.

Horng, S., Kreiman, G., Ellsworth, C., Page, D., Blank, M., Millen, K., and Sur, M. (2009). Differential gene expression in the developing lateral geniculate nucleus and medial geniculate nucleus reveals novel roles for Zic4 and Foxp2 in visual and auditory pathway development. J. Neurosci. 29, 13672-13683.

$\mathrm{Hu}$, B. (2003). Functional organization of lemniscal and nonlemniscal auditory thalamus. Exp. Brain Res. 153, 543-549.

Imig, T. J., and Morel, A. (1983). Organization of the thalamocortical 
auditory system in the cat. Annu. Rev. Neurosci. 6, 95-120.

Kapfer, C., Seidl, A. H., Schweizer, H., and Grothe, B. (2002). Experiencedependent refinement of inhibitory inputs to auditory coincidencedetector neurons. Nat. Neurosci. 5, 247-253.

Katoh, Y. Y., and Benedek, G. (1995). Organization of the colliculosuprageniculate pathway in the cat: A wheat germ agglutinin-horseradish peroxidase study. J. Comp. Neurol. 352, 381-397.

Kim, G., and Kandler, K. (2003). Elimination and strengthening of glycinergic/GABAergic connections during tonotopic map formation. Nat. Neurosci. 6, 282-290.

Kobler, J. B., Isbey, S. F., and Casseday, J. H. (1987). Auditory pathways to the frontal cortex of the mustache bat, Pteronotus parnellii. Science 236, 824-826.

Leake, P. A., Hradek, G. T., Chair, L., and Snyder, R. L. (2006). Neonatal deafness results in degraded topographic specificity of auditory nerve projections to the cochlear nucleus in cats. J. Comp. Neurol. 497, 13-31.

Leake, P. A., Snyder, R. L., and Hradek, G. T. (2002). Postnatal refinement of auditory nerve projections to the cochlear nucleus in cats. J. Comp. Neurol. 448, 6-27.

Lee, C. C., Imaizumi, K., Schreiner, C. E., and Winer, J. A. (2004). Concurrent tonotopic processing streams in auditory cortex. Cereb. Cortex 14, 441-451.

Luethke, L. E., Krubitzer, L. A., and Kaas, J. H. (1989). Connections of primary auditory cortex in the New World monkey, Saguinus. J. Comp. Neurol. 285, 487-513.

Malinowska, M., and Kosmal, A. (2003). Connections of the posterior thalamic region with the auditory ectosylvian cortex in the dog. J. Comp. Neurol. 467, 185-206.
Morel, A., and Imig, T. J. (1987). Thalamic projections to fields A, Al, P and VP in cat auditory cortex. J. Comp. Neurol. 265, 119-144.

Morel, A., and Kaas, J. H. (1992). Subdivisions and connections of auditory cortex in owl monkeys. J. Comp. Neurol. 318, 27-63.

Pallas, S. L., Roe, A. W., and Sur, M. (1990). Visual projections induced into the auditory pathway of ferrets. I. Novel inputs to primary auditory cortex (AI) from the LP/pulvinar complex and the topography of the MGN-AI projection. J. Comp. Neurol. 298, 50-68.

Pearson, J. M., Crocker, W. D., and Fitzpatrick, D.C. (2007). Connections of functional areas in the mustached bat's auditory cortex with the auditory thalamus. J. Comp. Neurol. 500, 401-418.

Raczkowski, D., Diamond, I. T., and Winer, J. A. (1976). Organization of thalamocortical auditory system in the cat studied with horseradish peroxidase. Brain Res. 101, 345-354.

Radtke-Schuller, S., Schuller, G., and O'Neill, W. E. (2004). Thalamic projections to the auditory cortex in the rufous horseshoe bat (Rhinolophys rouxi). II. Dorsal fields. Anat. Embryol. 209, 77-79.

Razak, K. A., and Fuzessery, Z. M. (2002). Functional organization of the pallid bat auditory cortex: Emphasis on binural organization. J. Neurophysiol. 87, 72-86.

Razak, K. A., and Fuzessery, Z. M. (2007). Development of functional organization of the pallid bat auditory cortex. Hearing Res. 228, 69-81.

Razak, K.A., Fuzessery, Z.M., and Lohuis, T. D. (1999). Single cortical neurons serve both echolocation and passive sound localization. J. Neurophysiol. 81, 1438-1442.

Razak, K. A., Richardson, M. D., and Fuzessery, Z. M. (2008). Experience is required for the maintenance and refinement of FM sweep selectivity in the developing auditory cortex. Proc. Natl. Acad. Sci. U.S.A. 105, 4465-4470.

Razak, K. A., Shen, W., Zumsteg, T., and Fuzessery, Z. M. (2007). Parallel thalamocortical pathways for echolocation and passive sound localization in a gleaning bat, Antrozous pallidus. $J$. Comp. Neurol. 500, 322-338.

Razak, K. A., Zumsteg, T., and Fuzessery, Z. M. (2009). Development of auditory thalamocortical connections in the pallid bat, Antrozous pallidus. J. Comp. Neurol. 515, 231-242.

Read, H. L., Winer, J. A., and Schreiner, C. E. (2002). Functional architecture of auditory cortex. Curr. Opin. Neurobiol. 12, 433-440.

Roger, M., and Arnault, P. (1989). Anatomical study of the connections of the primary auditory area in the rat. J. Comp. Neurol. 287, 339-356.

Radtke-Schuller, S. (2004). Cytoarchitecture of the medial geniculate body and thalamic projections to the auditory cortex in the rufous horseshoe bat (Rhinolophus rouxi). I. Temporal fields. Anat. Embryol. 209, 59-76.

Radtke-Schuller, S., Schuller, G., and O'Neill, W. E. (2004). Thalamic projections to the auditory cortex in the rufous horseshoe bat (Rhinolophus rouxi). II. Dorsal fields. Anat. Embryol. 209, 77-91.

Rouiller, E.M.(1997). "Functional organization of the auditory pathways," in The Central Auditory System, eds G. Ehret and R. Romand (New York: Oxford University Press), 3-96.

Rubsamen, R., Neuweiler, G., and Sripathi, K. (1988). Comparative collicular tonotopy in two bat species adapted to movement detection, Hipposideros speoris and Megaderma lyra. J. Comp. Physiol. A 163, 271-285.

Suga, N. (1989). Principles of auditory information-processing derived from neuroethology. J. Exp. Biol. 146, 277-286.
Tanaka, K., Otani, K., Tokunaga, A. and Sugita, S. (1985). The reciprocal connections of the suprageniculate nucleus and the superior colliculus in the rat. Neurosci. Res. 3, 79-85.

Wepsic, J. G. (1966). Multimodal sensory activation of cells in the magnocellular medial geniculate nucleus. Exp. Neurol. 15, 299-318.

Wenstrup, J. J. (1999). Frequency organization and responses to complex sounds in the medial geniculate body of the mustached bat. J. Neurophysiol. 82, 2528-2544.

Wenstrup, J. J., Larue, D. T., and Winer, J. A. (1994). Projections of physiologically defined subdivisions of the inferior colliculus in the mustached bat: Targets in the medial geniculate body and extrathalamic nuclei. J. Comp. Neurol. 346, 207-236.

Winer, J. A., Miller, L. M., Lee, C. C., and Schreiner, C. E. (2005). Auditory thalamocortical transformation: structure and function. Trends Neurosci. 28, 255-263.

Conflict of Interest Statement: The authors declare that research was conducted in the absence of any commercial or financial relationships that could be construed as potential conflict of interest.

Received: 15 January 2010; paper pending published: 03 August 2010; accepted: 29 August 2010; published online: 21 September 2010.

Citation: Razak KA and Fuzessery ZM (2010) Development of parallel auditory thalamocortical pathways for two different behaviors. Front. Neuroanat. 4:134. doi: 10.3389/fnana.2010.00134 Copyright (C) 2010 Razakand Fuzessery. This is an open-access article subject to an exclusive license agreement between the authors and the Frontiers Research Foundation, which permits unrestricted use, distribution, and reproduction in any medium, provided the original authors and source are credited. 